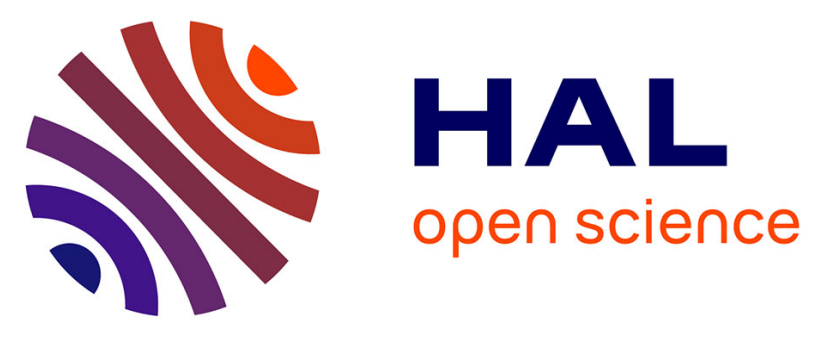

\title{
Zwischen Marketing und Mindset. Zur Rolle von Medien und grenzüberschreitender Berichterstattung im Kontext der Frankreichstrategie
}

\author{
Christoph Vatter
}

\begin{abstract}
To cite this version:
Christoph Vatter. Zwischen Marketing und Mindset. Zur Rolle von Medien und grenzüberschreitender Berichterstattung im Kontext der Frankreichstrategie. Hans-Jürgen Lüsebrink; Claudia PolzinHaumann; Christoph Vatter. "Alles Frankreich oder was ¿̨" - Die saarländische Frankreichstrategie im europäischen Kontext / "La France à toutes les sauces ¿" - La "Stratégie France" de la Sarre dans le contexte européen. Interdisziplinäre Zugänge und kritische Perspektiven / Approches interdisciplinaires et perspectives critiques., Transcript, pp.193-207, 2017, 9783839437551. 10.14361/9783839437551013. hal-03016424
\end{abstract}

\section{HAL Id: hal-03016424 \\ https://hal.univ-lorraine.fr/hal-03016424}

Submitted on 27 Nov 2020

HAL is a multi-disciplinary open access archive for the deposit and dissemination of scientific research documents, whether they are published or not. The documents may come from teaching and research institutions in France or abroad, or from public or private research centers.
L'archive ouverte pluridisciplinaire HAL, est destinée au dépôt et à la diffusion de documents scientifiques de niveau recherche, publiés ou non, émanant des établissements d'enseignement et de recherche français ou étrangers, des laboratoires publics ou privés.

\section{(1) (1) $\$$}

Distributed under a Creative Commons Attribution - NonCommercial - NoDerivatives 44.0 


\section{Zwischen Marketing und Mindset \\ Zur Rolle von Medien und grenzüberschreitender Berichterstattung im Kontext der Frankreichstrategie}

Avec la proclamation de la 'Stratégie France' en janvier 2014, le gouvernement du Land de la Sarre a lancé un véritable coup médiatique national et international. La 'Stratégie France' participe donc activement à la création d'une image de marque régionale de la Sarre dans la perspective d'un 'place branding'. Le rôle des médias est aussi essentiel pour réaliser les objectifs de la stratégie et pour ancrer une perspective franco-allemande dans le quotidien des Sarrois, notamment par l'établissement d'un espace public médiatique transfrontalier et interrégional. Dans les médias de la Grande Région, une telle orientation est certainement en émergence, mais encore assez.peu développée.

Les réflexions présentées dans cette contribution sont inspirées d'une rencontre-débat autour de deux ouvrages portant sur les cadres de la vie quotidienne et sur les constructions politiques dans la Grande Région SaarLorLux ainsi que sur la dimension médiatique de cet espace transfrontalier. ${ }^{1}$

Die Verkündung der Frankreichstrategie der Saarländischen Landesregierung im Januar 2014 kann als eine auf den ersten Blick recht erfolgreiche Marketingmaßnahme betrachtet werden, die breite Beachtung fand. Innerhalb von 30 Jahren soll das Saarland zu einem ,multilingualen Raum deutsch-französischer Prägung“"2 werden, sodass 2043 das Französische gleichsam als Zweitsprache in dem kleinen Bundesland im Dreiländereck etabliert wäre. Das Saarland, das sich schon zuvor als „französischstes aller Bundesländer“"3 bezeichnete, möchte dazu nicht nur das Erlernen der Sprache des Nachbarn weiter stärken, sondern damit auch seine „Frankreichkompetenz" ${ }^{\text {" }}$ weiter ausbauen.

1 Goulet, Vincent/Vatter, Christoph (Hg.): Gren₹überschreitende Informationsfliusse und Medien in der Großregion SaarLorLux/La circulation transfrontalière des informations médiatiques dans la Grande Région SaarLorLux, Baden-Baden: Nomos, 2015; Wille, Christian (Hg.): Lebenswirklichkeiten und politische Konstruktionen in Grensregionen. Das Beispiel der Großregion SaarLorLux: WirtschaftPolitik - Alltag - Kultur, Bielefeld: transcript, 2015.

2 Staatskanzlei des Saarlandes: Eckpunkte einer Frankreichstrategie für das Saarland, 2014, http:// www.saarland.de/dokumente/res_stk/D_Eckpunkte_Frankreich-Strategie_210114.pdf (31.01.2017), S. 9.

3 Vgl. Schönwald, Antje: Identitäten und Stereotype in grenzüberscbreitenden Verflechtungsräumen. Das Beispiel der Großregion, Wiesbaden: VS, 2012, S. 108.

4 Staatskanzlei des Saarlandes: Eckpunkte einer Frankreichstrategie, S. 1. 
Im zentralen Eckpunktepapier zur Frankreichstrategie ist die Rede von einer „Stärkung der Frankreichkompetenz im Land“ als Binnen-Strategie, und einer „Außen- und Kommunikationsstrategie“, die eben diese saarländische Frankreichkompetenz außerhalb des Saarlandes vermitteln soll. ${ }^{5}$ Einerseits gelang es dem kleinen Bundesland mit der Initiative der Frankreichstrategie in der Tat eine große mediale Aufmerksamkeit über seine Grenzen hinaus zu erreichen, sowohl in den nationalen als auch internationalen Medien; andererseits ist damit auch ein an die verschiedensten saarländischen Akteure adressiertes Programm zur Bündelung und Verstärkung zahlreicher auf Frankreich und das Erlernen der französischen Sprache sowie die grenzüberschreitende Zusammenarbeit ausgerichtete Initiativen verbunden.

Während die Außenwirkung also im Sinne eines, regional branding ${ }^{6}$ die ,Marke' Saarland mit neuer Orientierung auf die Grenzlage und damit verbundene kulturelle und sprachliche Kompetenzen mit frischem Elan aufwerten soll, kann unter der Binnenperspektive in erster Linie der Versuch der Stärkung und Motivierung der Akteure der deutsch-französischen Zusammenarbeit sowie der Förderung eines Verständnisses der Grenzlage und deutsch-französischen Geschichte des Saarlandes als Stärke und Kompetenz im Sinne eines ,empowerment ${ }^{\natural}-$ verstanden werden. Hierfür spielen die Berichterstattung und (kritische) Begleitung des Vorhabens durch die Medien eine zentrale Rolle, insbesondere ist an die Saarbrücker Zeitung und den Saarländischen Rundfunk als wichtigste regionale Medien zu denken. ${ }^{6}$

Im Folgenden sollen einige Aspekte zur Rolle der Medien im Kontext der Frankreichstrategie des Saarlandes im Spannungsfeld zwischen der Außenwirkung und der Binnenperspektive der saarländischen Öffentlichkeit diskutiert werden. Dabei ist es zunächst erforderlich zu erörtern, wie eine mit der Frankreichstrategie des Saarlandes angestrebte ,Frankreichkompetenz' profiliert sein könnte. In einem zweiten Schritt sollen dann die Medien im Hinblick auf eine interkulturelle, deutsch-französische Dynamik im Sinne der Außenund Binnenperspektive Frankreichstrategie betrachtet werden.

5 Staatskanzlei des Saarlandes: Eckpunkte einer Frankreichstrategie, S. 3.

6 Zur Rolle der Medien vgl. Staatskanzlei des Saarlandes: Eckpunkte einer Frankreichstrategie, S. 26-28. 


\section{Frankreichkompetenz, interkulturelle Kompetenz und interregionale Kompetenz}

In den zentralen Publikationen und öffentlichen Reden der Saarländischen Landesregierung zur Frankreichstrategie wird deutlich, dass der Begriff ,Frankreichkompetenz' zwar eine zentrale Rolle spielt, dieser aber in unterschiedlicher Art und Weise dargestellt und an keiner Stelle eindeutig definiert wird. Das in diesem Kontext häufigste Verständnis ist das einer auf das Erlernen und Beherrschen der französischen Sprache bezogenen „Französischkompetenz ${ }^{\text {"67 }}$, die konsistent mit der erklärten Zielvorstellung eines mehrsprachigen, vornehmlich deutsch-französisch geprägten Raums und den zahlreichen Maßnahmen aus dem (vor-)schulischen Bereich ist. An anderen Stellen wird diese, vornehmlich auf Fremdsprachenkompetenzen bezogene Vorstellung jedoch erweitert: „Frankreich-Kompetenz beinhaltet nicht nur entsprechende Sprachkompetenzen, sondern auch die Vertiefung weiterer Kompetenzfelder, wie Fachkompetenz, Regionalkompetenz und interkulturelle Kompetenz." 8 Hieraus geht hervor, dass eine deutsch-französische Ausrichtung des Saarlandes nur unter Rückbezug auf die regionale Ebene der grenzüberschreitenden Zusammenarbeit mit französischen Partnern unmittelbar jenseits der Grenze, aber auch im Rahmen der Großregion SaarLorLux denkbar ist, zu der neben dem Saarland, Lothringen ${ }^{9}$ und Luxemburg auch die Region Wallonien mit der deutschsprachigen und der französischen Gemeinschaft Belgiens sowie Rheinland-Pfalz gehören.

Die Vielzahl verschiedener Ansätze, ,Frankreichkompetenz' und damit verknüpfte Kompetenzbereiche im Rahmen der Frankreichstrategie zu konzeptualisieren, ${ }^{10}$ lässt sich auch auf unterschiedliche Akteursebenen zurückführen, wie beispielsweise Aussagen wie die folgende belegen, die eindeutig auf die Landesregierung als übergeordnete Instanz mit Koordinations- und Leitungsfunktion verweist: „Frankreich-Kompetenz dokumentiert sich sowohl in der Fähigkeit, die in der Breite des Saarlandes vorhandenen Aktivitäten frankreichspezifischer Art zu bündeln, als auch Spitzenleistungen hervorzuheben." 11

Mit dieser übergeordneten institutionellen Ebene sind auch im Eckpunktepapier verwendete Metaphern wie „Brücke“ bzw. „Tor“ zu Frankreich,

7 Staatskanzlei des Saarlandes: Eckpunkte einer Frankreichstrategie, S. 5.

8 Staatskanzlei des Saarlandes: Eckpunkte einer Frankreichstrategie, S. 20.

9 Seit der am 1. Januar 2016 in Kraft getretenen französischen Territorialreform ist Lothringen Bestandteil der Region Grand Est, zu der auch das Elsass und Champagne-Ardenne gehören, sodass die interregionale Zusammenarbeit in diesem Kontext neu konfiguriert werden muss.

10 Vgl. auch den Beitrag von Hans-Jürgen Lüsebrink in diesem Band.

11 Staatskanzlei des Saarlandes: Eckpunkte einer Frankreichstrategie, S. 7. 
„Mittler“ oder auch - in die Gegenrichtung gedacht - ,,Türöffner“ zu Deutschland" verknüpft. ${ }^{12}$ In zahlreichen weiteren Punkten verweist die Frankreichstrategie dagegen auf zivilgeschichtliche Akteure von Schulen über Vereine bis zum einzelnen Bürger, deren jeweilige Rollen auch durch jeweils spezifische Kompetenzprofile beschrieben werden könnten. Kenntnisse über Frankreich, die französische Kultur oder auch Spezifika im französischen Kommunikationsverhalten, Sprachkenntnisse und interkulturelle Handlungskompetenzen sind - um nur einige Beispiele zu nennen - eher auf einer individuellen Ebene angesiedelt und können dem Einzelnen helfen, erfolgreich mit französischen Gesprächspartnern zu kommunizieren.

Eine interkulturelle Frankreichkompetenz könnte demnach als Fähigkeit definiert werden, mit französischen Partnern auf der Grundlage von kulturellen und sprachlichen Kenntnissen in angemessener, wertschätzender und konstruktiver Art und Weise zu kommunizieren und zu interagieren. Sie ist in erster Linie als eine Transferkompetenz zu verstehen, da sie im Kern darauf abzielt, individuelle, soziale, fachliche und strategische Kompetenzen in ihrer bestmöglichen Kombination auf interkulturelle, deutsch-französische Handlungskontexte zu übertragen. ${ }^{13}$ Hierzu sind neben Sprachkenntnissen auch landeskundliche und kulturelle Kompetenzen notwendig. Im Rahmen der Frankreichstrategie für das Saarland kann eine so verstandene, deutsch-französisch profilierte interkulturelle Kompetenz nur als aus der spezifischen historischen Erfahrung der Region gewachsen und auf die lange erprobte interregionale Zusammenarbeit und das Zusammenleben in der Großregion bezogen verstanden werden. Auf der individuellen Ebene bezieht dies die Möglichkeit einer grenzüberschreitenden Alltagspraxis - vom Einkaufen bis zum Besuch von Kulturveranstaltungen oder Sport - mit ein; und auch auf der institutionellen Ebene können die langjährigen Erfahrungen aus der grenzüberschreitenden Zusammenarbeit in vielfältigen Bereichen für eine übergreifende, deutsch-französische (nationale) Ebene fruchtbar gemacht werden, wie beispielsweise die Vertretung des Saarlandes in Paris oder die Ansiedlung deutsch-französischer Institutionen wie die Deutsch-Französische Hochschule ${ }^{14}$ zeigen.

Interkulturelle Frankreichkompetenz im saarländischen Kontext muss also auf der Erfahrung der grenzüberschreitenden regionalen Zusammenarbeit und Alltagspraxis aufbauen. Diese ist jedoch keineswegs eine Selbst-

12 Staatskanzlei des Saarlandes: Eckpunkte einer Frankereichstrategie, S. 13.

13 Vgl. Bolten, Jürgen: Interkulturelle Kompeten₹, Erfurt: Landeszentrale für politische Bildung, 52012, S. 126-130.

14 Vgl. den Beitrag von Patricia Oster-Stierle in diesem Band. 
verständlichkeit, die für das gesamte Bundesland gilt. Wie Studien zur Großregion SaarLorLux zeigen, besteht diese aus einem „funktionalen Kern"15 entlang der deutsch-französischen Grenze sowie um Luxemburg, während für die Bewohner weiter entfernt liegender Gegenden die Nachbarn jenseits der Grenze eine weitaus geringere Rolle spielen und kaum Bestandteil alltäglicher Praktiken sind. Ein zentraler Ansatzpunkt in der Umsetzung der Frankreichstrategie stellt also die Förderung eines, interregionalen Mindsets' dar, d. h. die Verankerung einer grenzüberschreitenden, mehrsprachigen Realität in den Mentalitäten der Menschen, sodass diese selbstverständlich ,mitgedacht ${ }^{\star}$ wird - auch von den Saarländerinnen und Saarländern, die nicht entlang des Korridors der deutsch-französischen nationalen Grenze leben. Für die Ausbildung eines solchen interregionalen Mindsets, das nicht nur als eigene „Regionalkompetenz"16 für die Großregion, sondern als zentrale Voraussetzung für eine interkulturelle Frankreichkompetenz zu verstehen ist, und seiner Verankerung in der Alltagspraxis und den Mentalitäten der Menschen spielen die Medien eine zentrale Rolle; aber auch die institutionelle Ebene der innerdeutschen, interregionalen und internationalen (Außen-)Beziehungen des Saarlandes ist auf die mediale Berichterstattung angewiesen, damit die Ambitionen der Frankreichstrategie erfüllt werden können. In den folgenden Abschnitten soll daher zunächst die Rolle der Medien für die Außenwirkung des Saarlandes näher beleuchtet werden, ehe wieder die Binnenperspektive der regionalen Medien und ihrer Vermittlungsfunktion im Sinne des genannten interregionalen, deutsch-französischen Mindsets in den Fokus rückt.

\section{Zur Rezeption der Frankreichstrategie außerhalb des Saarlandes}

Die Verkündung der Frankreichstrategie im Januar 2014 verhalf dem Saarland zu einer großen medialen Aufmerksamkeit. Fast alle nationalen und zahlreiche internationale Zeitungen berichteten über die Initiative. Der Ruf als ,französischstes Bundesland" schien sich weiter zu konsolidieren und auch ein Blick in die aktuellen Daten zum Fremdsprachenlernen in deutschen Schulen, veröffentlicht in der Studie Schulen auf einen Blick (2016), bestätigt dies: 59,7 \% der saarländischen Schülerinnen und Schüler lernten im Schuljahr 2014/15 Französisch, etwas weniger $(54,3 \%)$ die englische Sprache. ${ }^{17}$ Damit führt das

15 ESPON: METROBORDER. Grenæüberschreitende polyzentrische Metropolregionen. Zielgerichtete Analysen 2013/2/3. Abschlussbericht, Luxemburg: ESPON/University of Luxembourg, 31.12.2010, http://www.dat.public.lu/publications/documents/metroborder/metroborder_ final_report_de.pdf (31.01.2017).

16 Staatskanzlei des Saarlandes: Eckpunkte einer Frankreichstrategie, S. 20.

17 Statistisches Bundesamt (Hg.): Schulen auf einen Blick. 2016, Wiesbaden: Statistisches Bundesamt, 2016, S. 20. Die hohen Werte für das Französische gehen sicherlich auf die verstärkte 
Saarland die Rangliste an. Es folgen Baden-Württemberg (26,3 \%), Thüringen $(23,5 \%)$ und Rheinland-Pfalz $(23,4 \%)$, d. h. auch unter den unmittelbar an Frankreich angrenzenden Bundesländern nimmt das Saarland eine Spitzenposition ein - der Bundesdurchschnitt lag bei $18 \%$.

Diese Form von Öffentlichkeit und Führungsrolle in Bezug auf das Französische ist in der Frankreichstrategie fest verankert, denn diese ist als eine Ausprägung von Public Diplomacy zu verstehen, d. h. sie zielt darauf ab, das Saarland national wie international weiter bekannt zu machen, sein Image in der öffentlichen Wahrnehmung zu verbessern und mit der Frankreichstrategie als „Dachmarke“18 fest zu verbinden. Dies kann jedoch nur im Zusammenspiel mit den Medien erreicht werden, wie auch am Beispiel der im Saarland angesiedelten deutsch-französischen und französischen Institutionen im Eckpunktepapier der Landesregierung ausgeführt wird:

Eine noch bessere Wahrnehmung und Sichtbarkeit dieser Einrichtungen in ganz Deutschland, Frankreich und Europa würde dazu beitragen, dass das Land mit seiner FrankreichKompetenz noch ernster genommen würde. Dabei kommt den Medien eine wichtige Vermittlungsfunktion zu. ${ }^{19}$

Die Herausforderung besteht darin, die Frankreichstrategie und die damit verknüpfte Vorstellung einer saarländischen Frankreichkompetenz in ihrer Vielfalt zu vermitteln, damit auch politische und ökonomische Effekte wie z. B. neue Kooperationen für Wirtschaftsunternehmen oder auch die Steigerung der Attraktivität des Saarlandes als Wohnregion erzielt werden können. Diese Problematik wird bei einem Blick in die überregionale und internationale Berichterstattung deutlich. ${ }^{20}$ Denn hier dominiert zunächst die Idee

Förderung von Frühfranzösisch im Saarland zurück. Englisch ist fester Bestandteil der in der Frankreichstrategie verankerten Mehrsprachigkeitsperspektive, in der sowohl Französisch als auch Englisch einen festen Platz einnehmen. Die Zahlen dürften aber weniger als direkte Erfolge der Frankreichstrategie interpretiert werden, sondern vielmehr als Früchte von Vorarbeiten, v. a. im Rahmen des Sprachenkonzepts des Saarlandes von 2011, nach dem alle saarländischen Schüler Französisch und Englisch lernen sollen. Vgl. Ministerium für Bildung des Saarlandes: Sprachenkonzept Saarland 2011. Neue Wege zur Mehrsprachigkeit im Bildungssystem, Saarbrücken, 2011, http://www.saarland.de/dokumente/res_bildung/Das_ Sprachkonzept_Saarland_2011.pdf (31.01.2017).

18 Staatskanzlei des Saarlandes: Eckpunkte einer Frankreichstrategie, S. 3.

19 Staatskanzlei des Saarlandes: Eckpunkte einer Frankreichstrategie, S. 6.

20 Vgl. hierzu die Bachelorarbeit von Eichler, Steve: Die Rezeption der Frankreichstrategie des Saarlandes in deutschen und französischen Tageszeitungen in den Jabren 2014 und 2015, Universität des Saarlandes/Université de Lorraine, Bachelorarbeit, 2015. 
einer Zweisprachigkeit, die z. T. auch als gegen das Englische gerichtet interpretiert wird, ${ }^{21}$ was den Intentionen der Frankreichstrategie und des saarländischen Sprachenkonzepts völlig entgegenläuft. Insgesamt dominiert jedoch in der nationalen Berichterstattung im Januar 2014 eine kritische Analyse zwischen Standortmarketing und Kampf um die Überlebensfähigkeit des kleinen, durch die ,Schuldenbremse' stark belasteten Bundeslandes. In der französischen Presse werden diese Aspekte zwar durchaus angesprochen; zahlreiche Artikel beziehen sich aber auch auf die wirtschaftliche Dimension und unterstreichen die engen Verbindungen zwischen dem Saarland und Frankreich, aus denen sich neue Potenziale ergeben können. ${ }^{22}$ Diese Wahrnehmung spiegelt sich auch in einigen Initiativen wieder, für die die Kommunikation der Frankreichstrategie Anlass war, eine saarländische Delegation mit Vertretern aus Politik, Wirtschaft und Wissenschaft einzuladen, um Perspektiven der Kooperation im Sinne eines ,Tors nach Deutschland' auszuloten, z. B. aus den französischen Regionen Burgund und Normandie sowie dem frankophonen Nordafrika mit Tunesien. ${ }^{23}$

Trotz einer eher selektiv-verengten Wahrnehmung der Frankreichstrategie als Bilingualismus-Politik in vielen nationalen und internationalen Medien kann die damit verbundene Presse- und Öffentlichkeitsarbeit, die auch in französischer Sprache erfolgte und die sozialen Netzwerke Facebook und Twitter einbezieht, ${ }^{24}$ eine Reihe von Erfolgen verbuchen, v. a. durch die große Aufmerksamkeit, die dem Saarland in den überregionalen Medien Deutschlands zuteil wurde - und dies jenseits finanzpolitischer Debatten, sowie auch durch die internationale Wahrnehmung, insbesondere auf europäischer Ebene und in Frankreich.

21 Vgl. z. B. Höll, Susanne: Französisch? Nää merci, in: Süddentsche Zeitung, 30.01.2014, S. 6; Nonnenmacher, Günther: Die saarländische Vision, in: FAZ, 25.01.2014, S. 1.

22 Vgl. z. B. Madelin, Thibaut: La Sarre veut devenir un Land bilingue, in: Les Echos, 28.01.2014, S. 12; Le Tallec, Camille: La région allemande de la Sarre veut écrire son avenir en français, in: La Croix, 04.11.2014, S. 5.

23 Zum Potenzial der außereuropäischen Kooperation mit frankophonen Ländern vgl. den Beitrag von Sylvère Mbondobari in diesem Band.

24 Eine Analyse der Nutzung der sozialen Medien im Kontext der Frankreichstrategie bestätigt die zentrale Rolle der saarländischen Ministerpräsidentin - fast alle Tweets und Postings offizieller saarländischer Akteure zur Frankreichstrategie gehen von der Staatskanzlei aus; sie legt aber auch deutliche Defizite in der Kommunikation offen, wie z. B. die kaum genutzten interaktiven Möglichkeiten zur Vernetzung und Einbeziehung französischer Akteure. Vgl. hierzu Masterarbeit von Picart, Malika: La stratégie „France" $d u$ Land de Sarre: campagne d'information du gouvernement sarrois et impact dans la presse en France et en Allemagne, Universität des Saarlandes/Université de Lorraine, Masterarbeit, 2016. 
Dies wird insbesondere im Kontrast zur Deutschlandstrategie Lothringens deutlich. ${ }^{25}$ Zunächst nur im französischen Departement Moselle, dann auch in der Region Lorraine wurde als Reaktion auf die saarländische Initiative ein entsprechendes Strategiepapier erarbeitet, das im Sommer 2015 veröffentlicht wurde. Im Gegensatz zu den umfangreichen PR-Maßnahmen im Rahmen der Frankreichstrategie, wie z. B. Broschüren, Image-Kampagnen oder auch das Label „Partner der Frankreichstrategie“, zeichnet sich der Umgang mit der Deutschlandstrategie Lothringens eher durch Diskretion aus. Sogar das Strategiepapier selbst ist nur auf Umwegen zu finden. Diese mangelnde Sichtbarkeit ist allerdings sicherlich auch im Kontext der Neuordnung der französischen Regionen zu sehen, im Zuge derer Lothringen seit Januar 2016 gemeinsam mit dem Elsass und Champagne-Ardenne zur Region Grand Est vereinigt wurde.

Insgesamt kann die Außenwirkung der Frankreichstrategie zumindest für den unmittelbaren Kontext ihrer Verkündung eher positiv bewertet werden; inwiefern dies auch nachhaltig der Fall ist, bleibt abzuwarten. Für die Verankerung einer interkulturellen Frankreichkompetenz im Alltag der Saarländerinnen und Saarländer und die Etablierung einer grenzüberschreitenden, deutsch-französischen Perspektive in Form eines interregionalen Mindsets ist die überregionale und internationale Berichterstattung dagegen eher von mittelbarer Bedeutung, wenn die positive Außenwahrnehmung beispielsweise medial ins Saarland zurückgespiegelt wird.

\section{Frankreichstrategie und grenzüberschreitende Berichterstattung in den saarländischen Medien}

Die Meinungsforschung scheint der Frankreichstrategie einen starken Rückhalt in der saarländischen Bevölkerung zu bescheinigen. So sprachen sich in einer Umfrage des Saarländischen Rundfunks 67 \% der 1000 befragten Saarländerinnen und Saarländer für ein bilinguales Saarland aus, ${ }^{26}$ und auch eine Forsa-Umfrage im Auftrag des Magazins Forum im November 2016 kommt zu einem positiven Ergebnis: 70 \% Zustimmung zur Frankreichstrategie wird

25 Vgl. dazu ausführlich den Beitrag von Reiner Marcowitz in diesem Band. Die Deutschlandstrategie Lothringens wird außerdem in der Bachelorarbeit von Nadine Fischer untersucht, vgl. Fischer, Nadine: Regionale Strategien für das Partnerland? Die Deutschlandstrategie Lotbringens und die Frankereichstrategie des Saarlandes im Vergleich, Universität des Saarlandes, Bachelorarbeit, 2016.

26 Brenner, Ulrich: Zwei Drittel der Saarländer wollen eine zweisprachige Heimat, in: Saarbrücker Zeitung, 16.05.2014. 
berichtet. ${ }^{27}$ Ein genauerer Blick in die Befragung zeigt aber, dass nach dem Stellenwert des Französischunterrichts als erste Fremdsprache im Saarland gefragt wurde, was in der Tat von einer großen Mehrheit unterstützt wird, nicht aber explizit nach dem politischen Projekt Frankreichstrategie. ${ }^{28}$ In der gleichen Umfrage bejahen aber auch $73 \%$, dass „eine stärkere Einbindung des Saarlandes in die Saar-Lor-Lux-Region für das Saarland konkrete Vorteile" bringt ${ }^{29}$ Dies bestätigt, dass einerseits das Erlernen der Nachbarsprache und die Bedeutung der Vernetzung in der Großregion als Basis für die breite Verankerung einer Frankreichkompetenz unverzichtbar sind.

Den regionalen Medien kommt hier eine besondere Rolle zu, wie auch das Eckpunktepapier zur Frankreichstrategie unterstreicht:

Hier tragen auch die regionalen Medien eine besondere Verantwortung. Nur wenige nutzen bislang konsequent die Möglichkeiten der grenzüberschreitenden Berichterstattung zum unmittelbaren Nutzen ihrer Zielgruppe im regionalen und nationalen Wettbewerb. ${ }^{30}$

In einem eng vernetzten, grenzüberschreitenden Raum, in dem die Grenze eher als Kontakt- und Begegnungszone gedacht wird, sollten die Medien also aus Sicht der Frankreichstrategie die deutsch-französische bzw. interregionale Dimension in der ganzen Breite ihrer Berichterstattung mit berücksichtigen.

Die saarländischen Medien begleiten die Frankreichstrategie intensiv. So zeigt beispielsweise eine Auswertung der Saarbrücker Zeitung, ${ }^{31}$ dass 2014 insgesamt 98 Artikel zur Frankreichstrategie publiziert wurden, davon 37 im ersten Quartal, also im Kontext der Verkündung der Initiative der saarländischen Landesregierung. Die Berichterstattung ging danach zwar zurück, die Thematik blieb aber beständig präsent. So wurden im vierten Quartal 2014 noch 18 Artikel veröffentlicht und auch im ersten Halbjahr 2015 finden sich noch 27 Berichte zum Thema. Diese intensive Berichterstattung ist in erster Linie darauf zurückzuführen, dass die Ministerpräsidentin Annegret Kramp-Karrenbauer die Frankreichstrategie zur, Chefsache` erklärte, sodass viele Politikfelder damit verknüpft wurden und sich häufig Anlässe zur Berichterstattung mit Bezug auf die Frankreichstrategie ergaben. Auch die zahlreichen Akteure der deutsch-französischen Zusammenarbeit im Saarland erhielten auf diese Weise einen privilegierten Zugang zur Öffentlichkeit und zu den Medien.

27 Vgl. z. B. die Meldung auf Radio Salü: Große Zustimmung zur Frankreichstrategie, 25.11.2016, www.salue.de/nachrichten/message.phtml?id=80859 (25.11.2016).

28 Vgl. Hilt, Oliver: Großer Stimmungstest zur Wahl, in: Forum. Das Wochenmagazin, 25.11.2016, http://www.magazin-forum.de/news/politik/gro\%C3\%9Fer-stimmungstestzur-wahl (31.01.2017).

29 Hilt: Großer Stimmungstest zur Wahl.

30 Staatskanzlei des Saarlandes: Eckpunkte einer Frankreichstrategie, S. 8.

31 Vgl. Eichler: Die Rezeption der Frankreichstrategie des Saarlandes. 
Während die Berichterstattung über die Frankreichstrategie in der saarländischen Presse also einen großen Stellenwert einnimmt, ist dies für die grenzüberschreitende und deutsch-französische Berichterstattung nur sehr bedingt der Fall, wie auch inhaltsanalytische Studien, Untersuchungen der grenzüberschreitenden Mediennetzwerke sowie Erkenntnisse zu interkulturellen Herausforderungen in der Großregion aufgrund unterschiedlicher Journalismuskulturen bestätigen. ${ }^{32}$

Eine Voraussetzung für die Verbreitung eines interregionalen Mindsets in der Bevölkerung stellt allerdings die Verwirklichung einer (medialen) Öffentlichkeit im Habermas'schen Sinne dar, die ebenfalls grenzüberschreitend-interregional aufgestellt ist und eine interkulturelle, deutsch-französische Dimension mit berücksichtigt. ${ }^{33}$

Eine solche interregionale (Medien-)Öffentlichkeit könnte - in Analogie zu Forschungen zu einer europäischen Öffentlichkeit - in struktureller Hinsicht auf zwei verschiedene Weisen aufgebaut sein. ${ }^{34}$ Erstens als gemeinsame, länderübergreifende Öffentlichkeit mit einer eng verzahnten, mehrsprachigen Medienlandschaft, in der Medien dies- und jenseits der Grenzen wechselseitig aufeinander Bezug nehmen. In diesem Modell würden auch die Menschen der Region ungeachtet ihres Wohnorts auf die gleichen Medien zugreifen, sodass diese als Foren für gesellschaftliche Debatten in einem gemeinsamen, mehrsprachigen und interkulturellen Raum dienten. Zweitens ist eine interregionale Öffentlichkeit über die Grenzen hinweg auch in Form von ,interregionalisierten' national-regionalen Öffentlichkeiten denkbar. Dieses Modell zeichnet sich dadurch aus, dass die Medien der jeweiligen Teilregionen - wie das Saarland, Lothringen oder auch Luxemburg - verstärkt Themen aus der Großregion aufgreifen, multiperspektivisch auf die Sichtweisen jenseits der Grenzen eingehen und es so erlauben, gesellschaftliche Debatten in einem grenzüberschreitenden, interregionalen Kontext zu führen.

32 Vgl. Goulet/Vatter: Grenzüberschreitende Informationsflüsse; Goulet, Vincent/Vatter, Christoph: L'espace médiatique transfrontalier: médias, flux d'informations et pratiques journalistiques, in: Wille (Hg.): Lebenswirklichkeiten und politische Konstruktionen in Grenaregionen, S. 229-248.

33 In Ansätzen wird eine konsequente Einbeziehung der Realitäten jenseits der Grenzen am ehesten in der Luxemburger Gratispresse umgesetzt, die damit auf die Berufspendler nach Luxemburg als wichtiger Teil der Leserschaft reagiert. Vgl. Goulet/Vatter: L'espace médiatique transfrontalier.

34 Vgl. Gerhards, Jürgen: Europäisierung von Ökonomie und Politik und die Trägheit der Entstehung einer europäischen Öffentlichkeit, in: Bach, Maurizio (Hg.): Die Europäisierung nationaler Gesellscbaften, Wiesbaden: VS Verlag für Sozialwissenschaften, 2000, S. 277-305; Wimmel, Andreas: Transnationale Diskurse in Europa. Der Streit um den Türkei-Beitritt in Deutschland, Frankreich und Großbritannien, Frankfurt/M.: Campus, 2006. 
Mit Blick auf das Saarland und die Großregion ist eine Realisierung der ersten Option wenig wahrscheinlich - zu sehr fallen noch sprachliche Hürden, die nationalen und regionalen politischen Spezifika, ihre jeweiligen Journalismuskulturen und Medienlandschaften ins Gewicht. Die zweite Perspektive mit dominant nationalen bzw. regionalen Öffentlichkeiten, die allerdings im Sinne einer interregionalen, multiperspektivischen Öffnung operieren, erscheint dagegen realistisch und - mit etwas Optimismus - vielleicht sogar schon im Entstehen.

Trotz einiger positiver Tendenzen zu einer insgesamt steigenden Präsenz grenzüberschreitender Themen zeigt aber beispielsweise schon der Blick in die Rubriken des Online-Auftritts der Saarbrücker Zeitung, dass Grenzen auf der strukturellen Ebene noch wenig überschritten werden, sondern die traditionellen politischen und territorialen Strukturen deutlich überwiegen (Saarland, Regionalverband, Landkreise, Gemeinden...). Unter lokaler und regionaler Berichterstattung wird also in erster Linie das eigene Bundesland verstanden und eher punktuell über die Grenze geblickt, die noch eine stark trennende Wirkung zu haben scheint.

Dass jedoch eine Pauschalverurteilung der saarländischen Medien nicht angemessen ist, wird im Vergleich mit der Frankreichberichterstattung des Saarländischen Rundfunks (SR) deutlich. Frankreichkompetenz ist traditionell fest im Selbstverständnis des Senders verankert, das sich auch im Slogan „Grenzgänger aus Passion“/,passionnément frontalière“ und einer aktiven, zweisprachigen Kommunikation über die eigenen Frankreich bezogenen Aktivitäten ausdrückt. ${ }^{35}$ Neben dem Engagement im Bereich der frankophonen Musik - im Hörfunkprogramm wie auch als Veranstalter von Konzerten betrifft dies beispielsweise die Sportberichterstattung (Tour de France), Radiosendungen wie Kindernachrichten in französischer Sprache und auch eine Verankerung grenzüberschreitender, v. a. deutsch-französischer Berichterstattung im Fernsehprogramm. Darüber hinaus sind auf institutioneller Ebene die vom SR bereits 1963 mitbegründete Deutsch-Französische Hörfunkkommission sowie die Veranstaltung des Deutsch-Französischen Journalistenpreises zu nennen.

In den Zeitraum seit Ausrufung der Frankreichstrategie fallen beispielsweise die Einrichtung eines eigenen Online-Frankreichthemenportals ${ }^{36}$, eine feste Verankerung des grenzüberschreitenden Magazins „Grenzenlos“ im wöchentlichen Sendeschema als Teil von „Wir im Saarland“ seit Ende 2016 und eine Überarbeitung der Wetterkarte, die seit Herbst 2014 nicht mehr an

35 Vgl. Saarländischer Rundfunk: Gelebte Nacbbarschaft. Der SR als Medium und Faktor in der Großregion/La transfrontalité au quotidien. La Saarländischer Rundfunk média et facteur de la Grande Région, Saarbrücken, 2016, http://www.sr.de/sr/home/nachrichten/vis_a_vis/broschuere_ gelebte_nachbarschaft100.pdf (31.01.2017).

36 Saarländischer Rundfunk: VIS-A-VIS, http://www.sr.de/visavis (31.01.2017). 
der saarländischen Landesgrenze endet. Maßnahmen wie die letztgenannten sprechen für eine Verankerung der grenzüberschreitenden Berichterstattung in einem regionalen Alltagsgeschehen und können so einen wichtigen Beitrag für die Etablierung eines interregionalen Mindsets leisten.

Diese deutlichen Unterschiede zwischen Rundfunk und regionaler Tageszeitung müssen allerdings differenziert betrachtet werden und können nicht allein auf Aspekte wie ein mehr oder weniger ausgeprägtes grenzüberschreitendes Bewusstsein oder deutsch-französisches Engagement zurückgeführt werden. Diese stellen zwar eine unabdingbare Voraussetzung dafür dar, ${ }^{37}$ aber die Platzierung einer regionalen Tageszeitung und einer Rundfunkanstalt der ARD in der Medienlandschaft mit ihren spezifischen medialen und wirtschaftlichen Kontextbedingungen führt zu grundverschiedenen Voraussetzungen für ein Frankreichengagement. Denn der Saarländische Rundfunk kann Frankreichkompetenz auch für die eigene Positionierung innerhalb der Sendeanstalten der ARD nutzen. Damit steht der kleine Sender vor ähnlichen Herausforderungen wie das Saarland im Reigen der Bundesländer: Die grenzüberschreitende, deutsch-französische Orientierung und Frankreichkompetenz können als Standortvorteil genutzt werden und die Überlebensfähigkeit in der ARD sichern. Bei der Saarbrücker Zeitung handelt es sich demgegenüber um eine regionale Tageszeitung mit quasi Monopolcharakter, die nicht in einer direkten Konkurrenz zu anderen Regionen bzw. überregionalen Zeitungen steht. Sie muss daher auch die ,Frankreich-Karte' nicht unbedingt ausspielen, um sich von diesen abzugrenzen.

\section{Perspektiven}

Wie aufgezeigt, gibt es v. a. seit 2014 Tendenzen in den regionalen Medien, grenzüberschreitende Wirklichkeiten im Sinne eines interregionalen, deutschfranzösischen Mindsets zu integrieren, aber insbesondere in der Presse scheint man noch eher darauf zu warten, dass die Leser eine stärkere Verankerung auch über die Grenze hinweg einfordern. Um eine interkulturelle Frankreichkompetenz nachhaltig zu fördern und in der Breite zu verankern, könnten die saarländischen Medien (ebenso wie die jenseits der Grenze) einen wichtigen Beitrag leisten, indem sie die Grenze konsequent in ihrer Berichterstattung, Rubriken und Organisationsstrukturen mit-denken, über-denken und vielleicht auch manchmal weg-denken.

37 Vgl. Vatter, Christoph: Die Pressekonferenz in Deutschland und Frankreich - eine interkulturelle Herausforderung für die grenzüberschreitende Medienkommunikation? Journalistische Praktiken und Kommunikationsinstrumente am Beispiel der Großregion, in: Goulet/Vatter (Hg.): Grenzü̈berschreitende Informationsflïsse, S. 283-304. 
Als Anregung zur Förderung eines interregionalen Mindsets als Basis dafür, dass die Bevölkerung das Projekt Frankreichstrategie mit trägt und sich aktiv gestaltend einbringt, mag der soziologische Ansatz der Sozialintegration dienen, der zwar in erster Linie auf die Migrationsgesellschaft abzielt, der aber auch für die Integration neuer Ideen wie die Frankreichstrategie Denkanstöße vermitteln kann. Hartmut Esser unterscheidet vier Dimensionen der Sozialintegration: $:^{38}$ 1. Kulturation, d. h. der Erwerb von Wissen und Kompetenzen, beispielsweise zu typischen Situationen im Alltagshandeln oder gemeinsamen Wissensbeständen; 2. Platzierung, d. h. die Einnahme von gesellschaftlichen Funktionen durch die Akteure, wie z. B. bestimmte berufliche Positionen, woraus sich dann Möglichkeiten ergeben, um soziale Beziehungen untereinander zu pflegen; 3. Interaktion, d. h. verschiedene Beteiligte bauen Beziehungen zueinander auf, es entsteht ein Austausch über Wissensbestände und Symbole; 4. Identifikation, eine Einstellung, bei der Akteure sich als Teil des sozialen Gebildes betrachten und damit eine Einheit entsteht. Alle vier Dimensionen stehen in einem Wechselverhältnis, so ist z. B. eine gewisse Kulturation Voraussetzung für die Einnahme gesellschaftlicher Positionen (Platzierung), die wiederum Interaktion und Identifikation fördern kann.

Im Kontext der Frankreichstrategie und der Förderung eines deutschfranzösischen, interregionalen Bewusstseins im Alltag der saarländischen Bevölkerung, d. h. einer Identifikation im Sinne Essers, ist der Erwerb von Wissen und Kompetenzen (Kulturation) notwendig, damit grenzüberschreitende Beziehungen zwischen Menschen und Institutionen aufgebaut werden können (Platzierung und Interaktion). Sowohl für die Vermittlung von Wissen über den Anderen als auch die Identifikation als Teil einer gemeinsamen sozialen Einheit sind Medien mit grenzüberschreitender Berichterstattung und die Schaffung eines gemeinsamen Raums (Öffentlichkeit) für den gesellschaftlichen Austausch unabdingbar. Eine strukturelle Verankerung der grenzüberschreitenden Dimension in der Berichterstattung und eine interregional, deutsch-französisch ausgerichtete Grundeinstellung von Journalisten und Verantwortlichen der Medieninstitutionen stellen daher einen entscheidenden Schritt für eine nachhaltige Umsetzung der Frankreichstrategie des Saarlandes dar.

38 Vgl. Esser, Hartmut: Integration und ethnische Schichtung, in: Mannheimer Zentrum für Europäische Sozialforschung (Hg.): Arbeitspapiere 40 (2001), Mannheim: MZES, http:// www.mzes.uni-mannheim.de/publications/wp/wp-40.pdf (31.01.2017); Esser, Hartmut: Soziologie. Spezielle Grundlagen, Band 2: Die Konstruktion der Gesellschaft, Frankfurt/M. [u. a.]: Campus, 2000, insb. S. 272-275. 
Seit November 2014 werden Reisende auf der Autobahn an der Landesgrenze des Saarlandes mit Willkommensschildern mit der Aufschrift „Großes entsteht immer im Kleinen“ und auch der französischen Übersetzung „Les petits ruisseaux font les grandes rivières" begrüßt. ${ }^{39}$ Die Analyse der regionalen Medien zeigt, dass ein interregionales, deutsch-französisches Bewusstsein im geschilderten Sinne zumindest in Ansätzen bereits festzustellen ist. Seit Bekanntgabe der Frankreichstrategie sind diese Rinnsale grenzüberschreitender Berichterstattung stetig gewachsen; es ist ihnen zu wünschen, dass sie einmal zu großen Strömen werden. Das Potenzial dazu ist bereits sichtbar, aber es sind noch große Anstrengungen nötig, nicht nur auf dem Gebiet der Medien.

\section{Literaturverzeichnis}

Bolten, Jürgen: Interkulturelle Kompeten₹, Erfurt: Landeszentrale für politische Bildung, ${ }^{5} 2012$.

Brenner, Ulrich: Zwei Drittel der Saarländer wollen eine zweisprachige Heimat, in: Saarbrücker Zeitung, 16.05.2014.

Eichler, Steve: Die Rezeption der Frankreichstrategie des Saarlandes in deutschen und französischen Tageszeitungen in den Jabren 2014 und 2015, Universität des Saarlandes/Université de Lorraine, Bachelorarbeit, 2015.

Ernst, Nora: Imagepflege an der Grenze, in: Saarbrücker Zeitung, 29.11.2014.

ESPON: METROBORDER. Grenzüberschreitende polyzentrische Metropolregionen. Zielgerichtete Analysen 2013/2/3. Abschlussbericht, Luxemburg: ESPON/University of Luxembourg, 31.12.2010, http://www.dat.public.lu/publications/documents/metroborder/metroborder_ final_report_de.pdf (31.01.2017).

Esser, Hartmut: Soziologie. Spezielle Grundlagen, Band 2: Die Konstruktion der Gesellschaft, Frankfurt/M. [u. a.]: Campus, 2000.

Esser, Hartmut: Integration und ethnische Schichtung, in: Mannheimer Zentrum für Europäische Sozialforschung (Hg.): Arbeitspapiere 40 (2001), Mannheim: MZES, http://www. mzes.uni-mannheim.de/publications/wp/wp-40.pdf (31.01.2017).

Fischer, Nadine: Regionale Strategien für das Partnerland? Die Deutschlandstrategie Lotbringens und die Frankreichstrategie des Saarlandes im Vergleich, Universität des Saarlandes, Bachelorarbeit, 2016.

Gerhards, Jürgen: Europäisierung von Ökonomie und Politik und die Trägheit der Entstehung einer europäischen Öffentlichkeit, in: Bach, Maurizio (Hg.): Die Europäisierung nationaler Gesellschaften, Wiesbaden: VS Verlag für Sozialwissenschaften, 2000, S. 277-305.

Goulet, Vincent/Vatter, Christoph (Hg.): Grenzüberscbreitende Informationsflüsse und Medien in der Großregion SaarLorLux/La circulation transfrontaliere des informations médiatiques dans la Grande Région SaarLorLux, Baden-Baden: Nomos, 2015.

Goulet, Vincent/Vatter, Christoph: L'espace médiatique transfrontalier: médias, flux d'informations et pratiques journalistiques, in: Wille (Hg.): Lebenswirklichkeiten und politische Konstruktionen in Grensregionen, S. 229-248.

Hilt, Oliver: Großer Stimmungstest zur Wahl, in: Forum. Das Wochenmagazin, 25.11.2016, http://www.magazin-forum.de/news/politik/gro\%C3\%9Fer-stimmungstest-zur-wahl (31.01.2017).

39 Ernst, Nora: Imagepflege an der Grenze, in: Saarbrücker Zeitung, 29.11.2014. 
Höll, Susanne: Französisch? Nää merci, in: Süddeutsche Zeitung, 30.01.2014, S. 6.

Le Tallec, Camille: La région allemande de la Sarre veut écrire son avenir en français, in: $L a$ Croix, 04.11.2014, S. 5.

Madelin, Thibaut: La Sarre veut devenir un Land bilingue, in: Les Echos, 28.01.2014, S. 12.

Ministerium für Bildung des Saarlandes: Sprachenkonzept Saarland 2011. Neue Wege zur Mehrsprachigkeit im Bildungssystem, Saarbrücken, 2011, http://www.saarland.de/dokumente/res_ bildung/Das_Sprachkonzept_Saarland_2011.pdf (31.01.2017).

Nonnenmacher, Günther: Die saarländische Vision, in: FAZ, 25.01.2014, S. 1.

Picart, Malika: La stratégie „France“ $d u$ Land de Sarre: campagne d'information du gouvernement sarrois et impact dans la presse en France et en Allemagne., Universität des Saarlandes/Université de Lorraine, Masterarbeit, 2016.

Radio Salü: Große Zustimmung zur Frankreichstrategie, 25.11.2016, www.salue.de/nachrichten/ message.phtml?id=80859 (25.11.2016).

Saarländischer Rundfunk: Gelebte Nachbarschaft. Der SR als Medium und Faktor in der Großregion/La transfrontalité au quotidien. La Saarländischer Rundfunk média et facteur de la Grande Région, Saarbrücken, 2016, http://www.sr.de/sr/home/nachrichten/vis_a_vis/broschuere_gelebte_ nachbarschaft100.pdf (31.01.2017).

Saarländischer Rundfunk: VIS-A-VIS, http://www.sr.de/visavis (31.01.2017).

Staatskanzlei des Saarlandes: Eckpunkte einer Frankreichstrategie für das Saarland, 2014, http:// www.saarland.de/dokumente/res_stk/D_Eckpunkte_Frankreich-Strategie_210114.pdf (31.01.2017).

Statistisches Bundesamt (Hg.): Schulen auf einen Blick 2016, Wiesbaden: Statistisches Bundesamt, 2016.

Schönwald, Antje: Identitäten und Stereotype in grenæüberschreitenden Verflechtungsräumen. Das Beispiel der Großregion, Wiesbaden: VS, 2012.

Vatter, Christoph: Die Pressekonferenz in Deutschland und Frankreich - eine interkulturelle Herausforderung für die grenzüberschreitende Medienkommunikation? Journalistische Praktiken und Kommunikationsinstrumente am Beispiel der Großregion, in: Goulet/ Vatter (Hg.): Grenzüberscbreitende Informationsflüsse und Medien in der Großregion SaarLorLux, S. $283-304$.

Wille, Christian (Hg.): Lebenswirklichkeiten und politische Konstruktionen in Grenzregionen. Das Beispiel der Großregion SaarLorLux: Wirtschaft - Politik - Alltag - Kultur, Bielefeld: transcript, 2015.

Wimmel, Andreas: Transnationale Diskurse in Europa. Der Streit um den Türkei-Beitritt in Deutschland, Frankereich und Großbritannien, Frankfurt/M.: Campus, 2006. 